\title{
Dual-energy CT for the evaluation of silicone breast implants
}

\author{
Thorsten R. C. Johnson - Isabelle Himsl - Karin Hellerhoff • Doris Mayr • \\ Dorothea Rjosk-Dendorfer • Nina Ditsch • Bernhard Krauss • Klaus Friese • \\ Maximilian F. Reiser • Miriam S. Lenhard
}

\begin{abstract}
Objective The evaluation of breast implants for rupture is currently the domain of ultrasound and MRI, while mammography is of very limited diagnostic value. Recently, specific visualisation of silicone has become feasible using dual-energy CT. Our objective was to evaluate whether it is feasible to identify silicone in breast implants by dualenergy CT and to reliably diagnose or rule out ruptures. Methods Seven silicone breast implant specimens were examined on dual-source CT at 100- and $140-\mathrm{kV}$ tube potential with a $0.8-\mathrm{mm}$ tin filter (collimation $128 \times 0.6 \mathrm{~mm}$, current-time products 165 and $140 \mathrm{mAsref}$ with modulation, rotation time $0.28 \mathrm{~s}$, pitch 0.55 ). Two patients scheduled for implant removal or replacement were examined with identical parameters.

Results The silicone of the implant specimens showed a strong dual-energy signal. In one patient, both implants were intact, while a rupture was identified in the other patient. Ultrasound, MRI, surgical findings and histology confirmed the dual-energy CT diagnosis.
\end{abstract}

T. R. C. Johnson $(\bowtie) \cdot$ K. Hellerhoff • D. Rjosk-Dendorfer •

M. F. Reiser

Department of Clinical Radiology,

University of Munich-Grosshadern Campus,

Marchioninistr. 15,

81377 Munich, Germany

e-mail: thorsten.johnson@med.uni-muenchen.de

I. Himsl $•$ N. Ditsch $\cdot$ K. Friese $\cdot$ M. S. Lenhard Department of Obstetrics and Gynecology, University of Munich, Munich, Germany

D. Mayr

Department of Pathology, University of Munich,

Munich, Germany

B. Krauss

Siemens AG, Healthcare Sector,

Erlangen, Germany
Conclusion Dual-energy CT may serve as an alternative technique for speedy evaluation of silicone breast implants. Specific clinical studies are required to determine the diagnostic accuracy and define indications for this technique.

Key Points

- Dual-energy CT makes it possible to visualise silicone in breast implants.

- Silicone provides a strong photoelectric effect that can be detected.

- Initial experience suggests that implant ruptures can be identified or ruled out.

Keywords Dual-energy CT · Breast implants · Augmentation $\cdot$ Silicone $\cdot$ Implant rupture

\section{Introduction}

Breast augmentation is common throughout the world, be it for cosmetic or reconstructive purposes. Silicone and saline implants are the most common type of augmentation, and both represent a challenge for imaging. Recently, the problems concerning the French manufacturer PIP (Poly Implant Prothèse, La Seyne-sur-Mer, France), which used low-grade industrial silicone for most of their implants, sparked an increase in demand for medical imaging to evaluate breast implants in many countries [1]. French health authorities recommended close follow-up at 6-month intervals for all patients with intact PIP implants and immediate removal of ruptured implants [2].

The most frequent and relevant diagnostic question in this context is implant integrity. After implantation, the body physiologically builds a layer of fibrous tissue around the implant as a physiological reaction to the foreign material, forming a fibrous capsule [3]. Implant rupture can be classified as intracapsular or extracapsular, depending on the 
location of the escaped silicone with respect to this fibrous capsule. Most implant ruptures remain intracapsular. The median life expectancy of normal quality silicone implants is around 10-16 years and is related to the thickness and quality of the elastomer shell [4]. Another aspect of leakage is 'gel bleed', which represents a transudation of microscopic amounts of silicone gel through an intact shell. Recent studies revealed that PIP prostheses rupture and bleed more frequently [5-7] and that low-quality silicone gel can cause irritation and local inflammation, although there is no evidence of carcinogenic effects [8].

For cancer screening, mammography can be performed as implant-displaced projections (Eklund technique) in order to optimally visualise the glandular tissue anterior to the implant. However, silicone implants appear as dense oval masses that are too radiodense to allow a diagnostic evaluation using the $\mathrm{x}$-ray spectrum of mammography [9]. The rupture only becomes apparent if there is an extracapsular rupture and large conglomerates of radio-opaque silicone are seen in the glandular parenchyma or in axillary lymph nodes.

Therefore, the evaluation of implants is currently in the domain of ultrasound and MRI [9]. In ultrasound, the shell is visualised as single or double echogenic lines, and their continuity can be evaluated to exclude rupture. A reliable sign of intracapsular rupture is multiple curvilinear lines crossing through the interior of the implant, called the "stepladder" sign [10]. Also, in the case of extracapsular rupture, silicone conglomerates in the glandular parenchyma or in axillary lymph nodes can be recognised by increased echogenicity ("snowstorm sign") and strong posterior shadowing. The reported sensitivity and specificity of ultrasound for implant rupture are around 50-77\% and 55-84\%, respectively $[11,12]$.

The best diagnostic option for implant evaluation is MRI with a reported sensitivity of 74-100\% and specificity of 63$100 \%$, depending on the technique, with significant progress in MRI over the last years [4, 13-19] . Intracapsular rupture is recognised as the "linguine sign" based on hypointense lines of the shell in the bright signal of the silicone in T2-weighted sequences or the "salad oil" sign with round "drops" of altered signal from small inclusions of fluid within the silicone. Since recently, there are silicone-specific sequences using threepoint chemical-shift techniques [20] to highlight silicone in the glandular parenchyma or in axillary lymph nodes with
Fig. 1 Topogram showing seven different silicone breast implant specimens and one spacer [*] (a). CT images of a silicone implant obtained simultaneously at 100 (b) and Sn 140 kVp (c). Colour coding of the photoelectric effect shows homogeneous signal in the implant (d)
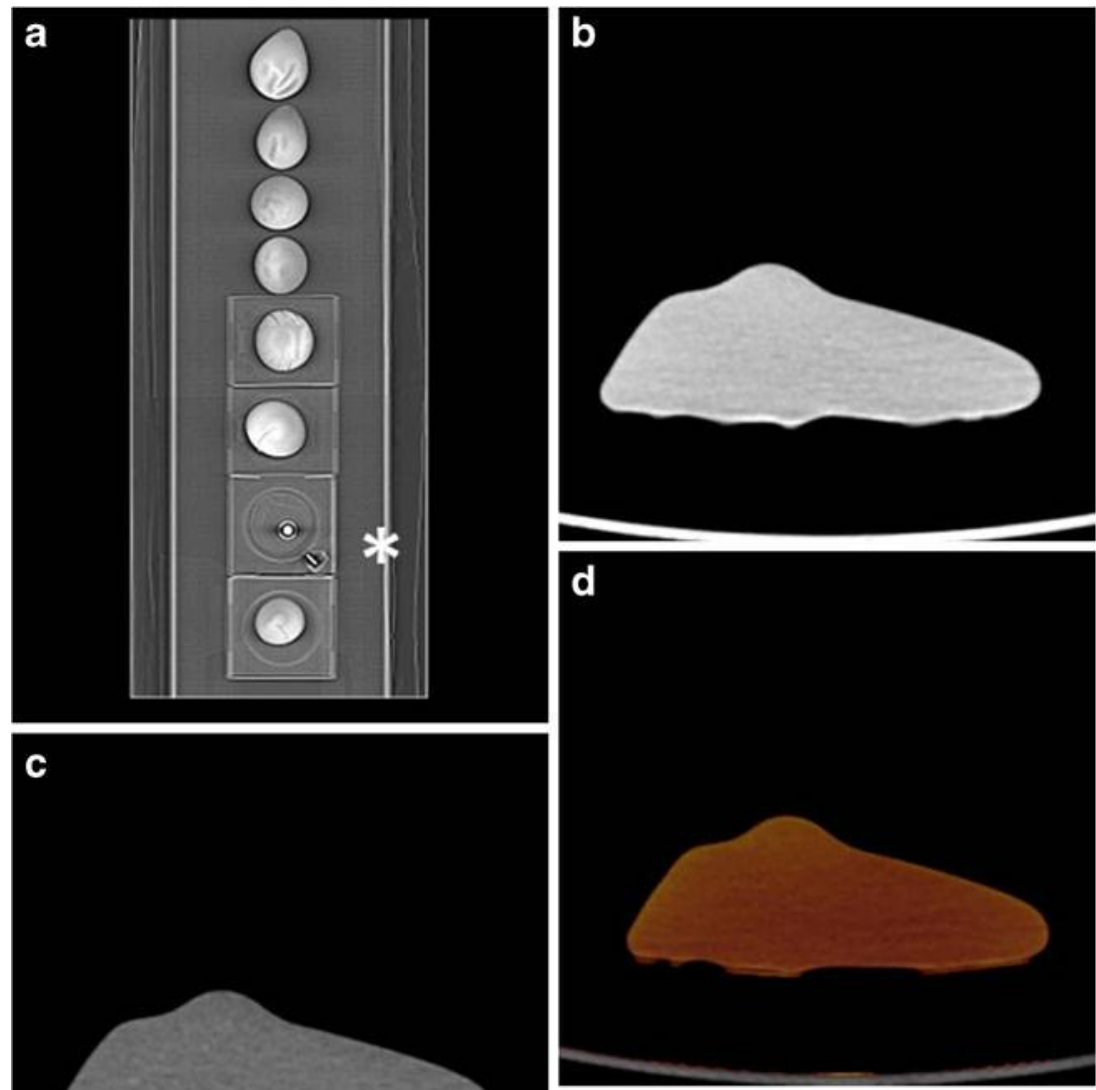

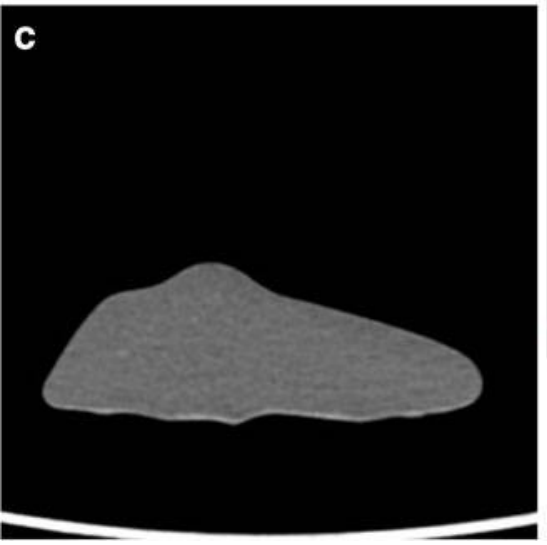



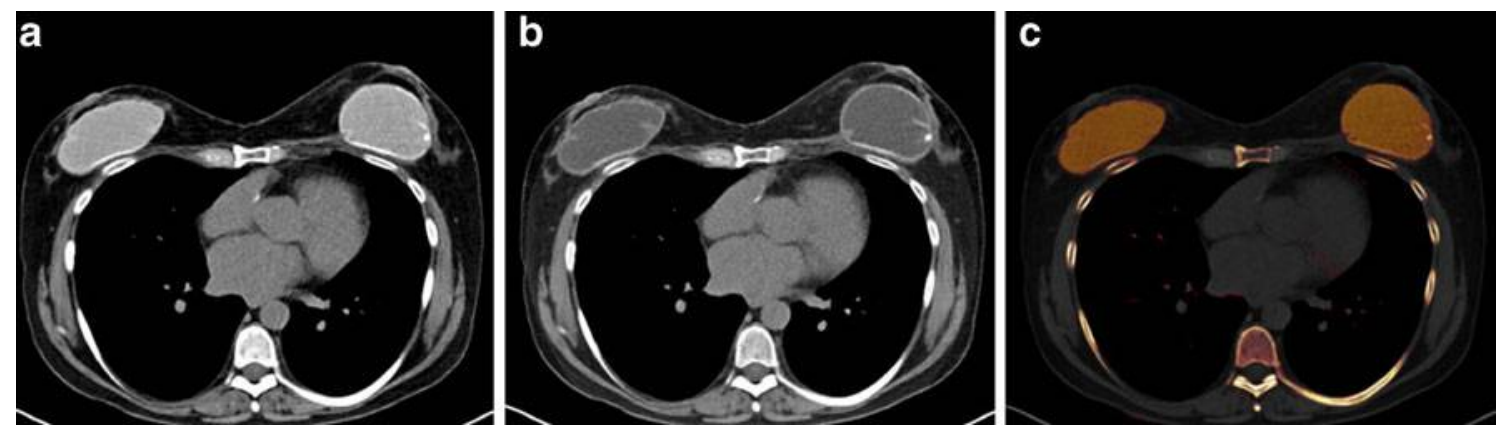

Fig. 2 A 48-year-old patient (patient A) examined for implant evaluation; CT images obtained simultaneously at 100 (a) and Sn $140 \mathrm{kVp}$ (b) and colour-coded silicone (c). There is strong signal from the silicone and no evidence of rupture

high signal, which are helpful for the detection of small extracapsular ruptures. On the other hand, there are specific diffusion-weighted sequences for cancer detection in implant patients suppressing the signal of both fat and silicone [21].

Although MRI represents the technique of choice, in some patients with contraindications, e.g. pacemakers, cochlear implants, or other metallic implants or foreign bodies, or in the case of severe claustrophobia, other diagnostic options would be desirable.

So far, CT has not played a role in the evaluation of the breast or implants. However, dual-energy CT has been clinically available since 2006 , offering the possibility of acquiring two CT data sets at the same time using different $\mathrm{x}$ ray spectra. The two data sets can be analysed voxel by voxel using a three-material decomposition algorithm in order to identify materials with photoelectric effect. Chemically, silicone or polymerised siloxanes consist of light atoms such as hydrogen, carbon, and oxygen, but also the metalloid element silicon with element number 14. Contrary to the lighter atoms, this element has a significant photoelectric effect, which can be visualised in dual-energy CT [22]. Therefore, the aim of this initial investigation was to evaluate whether it is feasible to identify silicone in breast implants by dual-energy CT and whether the image quality and contrast-to-noise ratio are sufficient to reliably identify or rule out ruptures. A secondary aim was to evaluate whether different types of silicone implants could be differentiated, because the imaging characteristics of PIP implants do not differ from those of other types of prostheses in other techniques [23] and many patients do not know which type of implant they have.

\section{Materials and methods}

Seven different silicone breast implants were examined using dual-source CT (Somatom Definition Flash, Siemens, Forchheim, Germany). Manufacturers of the prostheses were Mentor (Santa Barbara, CA, USA; three implants), Natrelle by Allergan (Marlow, Buckinghamshire, UK; two implants), Eurosilicone (Apt, France), and Poly Implat Prothèse (PIP, La Seyne-sur-Mer, France). The examination was performed using standard parameters for dual-energy $\mathrm{CT}$ of the chest, which are routinely used for lung perfusion imaging: voltages $140 \mathrm{kVp}$ with $0.8-\mathrm{mm}$ tin filter and $100 \mathrm{kVp}$; collimation $128 \times 0.6 \mathrm{~mm}$, reference tube current-time products (CareDose4D) 165 and $140 \mathrm{mAs}$, rotation time $0.28 \mathrm{~s}$ and pitch 0.55 . The implants were centred on the patient table in the gantry. The resulting images were analysed using the three-material decomposition algorithm
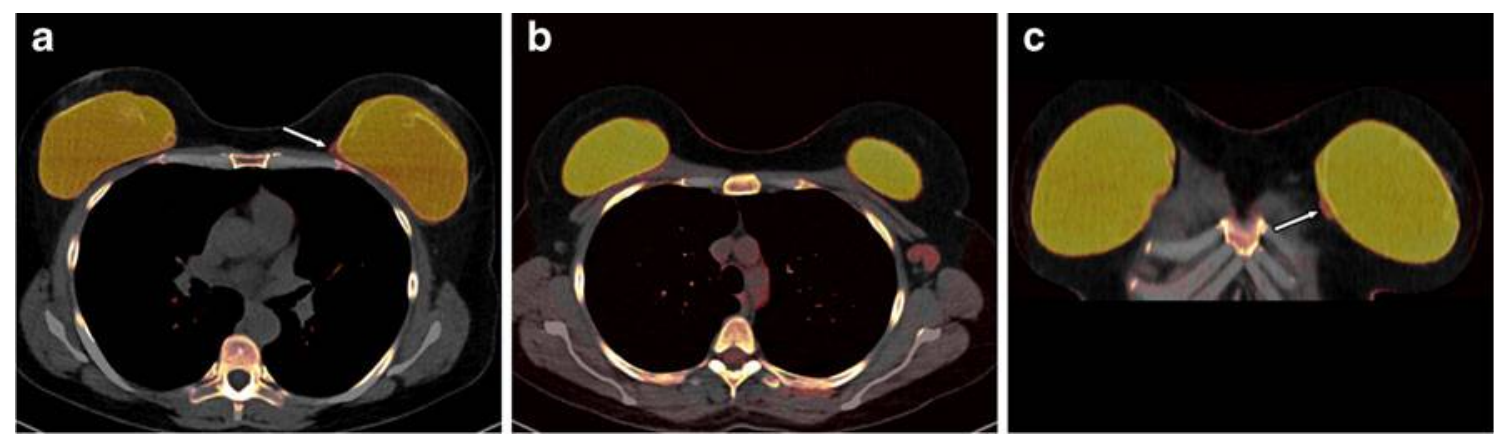

Fig. 3 A 51-year-old female patient (patient B) examined for the evaluation of PIP implants before surgical removal; CT images in axial (a, b) and coronal (c) orientation with colour-coded silicone signal.
Note the silicone extending outside the inferior medial shell of the left implant (arrows in $\mathbf{a}$ and $\mathbf{c}$ ) and the silicone signal in the enlarged axillary lymph node (b) 
Fig. 4 Ultrasound images of patient $\mathrm{B}$ showing a

"stepladder" sign of the implant (arrows in a) and increased echogenicity of the corresponding enlarged axillary lymph node (b)
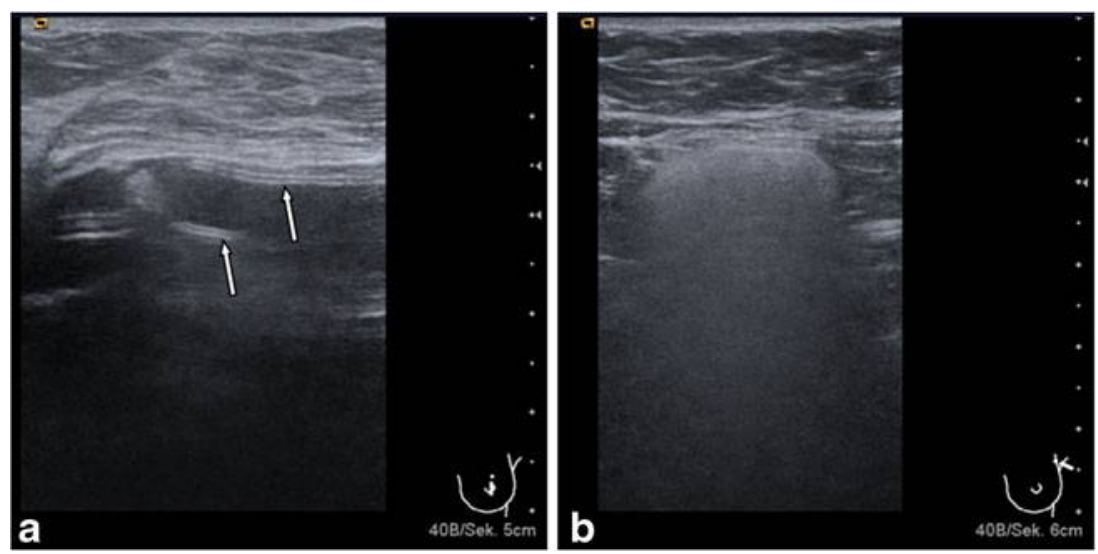

[22] of the dual-energy CT software (syngo VE32B) with the "Liver VNC" preset.

After the technical feasibility had been confirmed, two patients were examined. Both presented for breast implant evaluation before implant replacement or removal and were reluctant to undergo MRI owing to claustrophobia. Written informed consent was obtained before the examination after the nature of the procedure had been fully explained. One of the patients (patient A, 48 years old) had Mentor implants on both sides, which appeared intact on ultrasound. The other 51-year-old patient (patient B) had PIP implants, one of which had already been replaced with a new PIP implant. Ultrasound had shown signs of implant rupture in this patient.

The same parameters as mentioned above were used for the patient examinations. The patient table was positioned rather low in the gantry in order to ensure that the implants were entirely covered by the 33- $\mathrm{cm}$ field of view of the smaller detector. The examination range was limited to include the implants and axillary lymph nodes. No contrast material was applied. Examination times were 3 and $4 \mathrm{~s}$. The CT dose index was $7.7 \mathrm{mGy}$ and $8.1 \mathrm{mGy}$, the DLP 132 and $158 \mathrm{mGy}^{*} \mathrm{~cm}$, converting to equivalent doses of 2.2 and $2.7 \mathrm{mSv}$ [24].

\section{Results}

The implants showed a strong dual-energy signal of both the silicone inside the implant and the elastomer shell with a contrast-to-noise ratio of $8 \pm 1$ (Fig. 1). There were small differences in the individual density values of the specimen implants (ranges $115-131 \mathrm{HU}$ at $100 \mathrm{kVp}$ vs. 7-21 $\mathrm{HU}$ at $\mathrm{Sn} 140 \mathrm{kVp}$ ). There were also slight differences in density in different areas of the same implant, depending on the configuration and resulting differences in beam hardening and cupping artefacts. Thus, the differences in density between the prostheses of different suppliers were not significant and would not allow a differentiation.

In the patient examinations, the density values in both patients were equivalent $(104 \mathrm{HU}$ at $100 \mathrm{kVp} / 6 \mathrm{HU}$ at Sn140kVp). Colour coding of the silicone highlighted the implants with strong contrast and very weak noise in surrounding tissue. In patient $\mathrm{A}$, the silicone signal was confined to the envelope of the implants (Fig. 2). In patient B with the PIP prostheses, an extracapsular rupture was identified in the posterior lower inner aspect of the left implant (Fig. 3). The shell also showed multiple folds and layers crossing the silicone inside, suggesting a rupture and collapse of the shell.
Fig. 5 MRI of patient B showing a "salad oil" sign in the left implant on T2-weighted sequences (arrows in a) and increased signal of the enlarged left axillary lymph node on silicone-specific sequences (b)
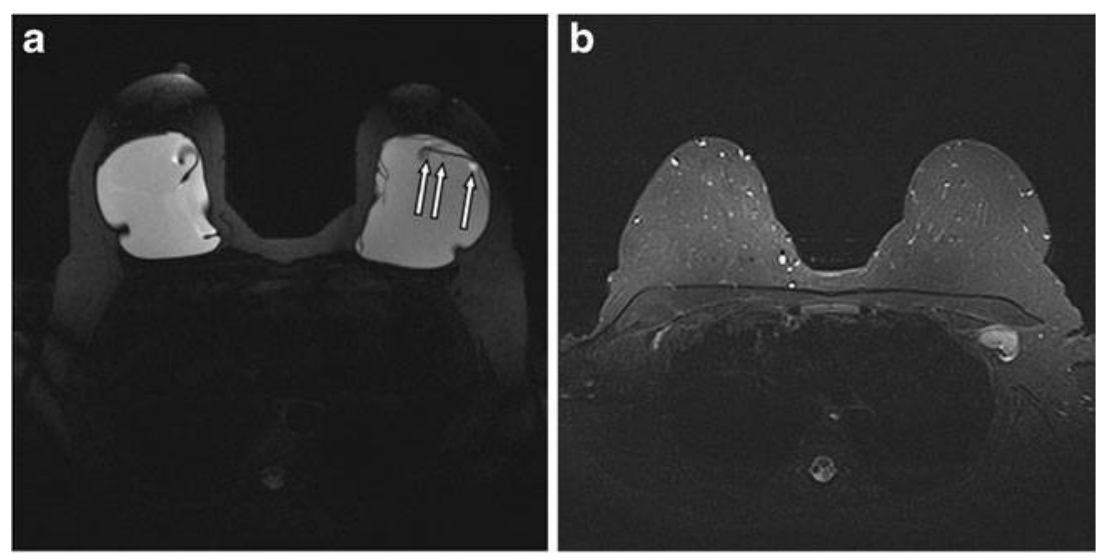

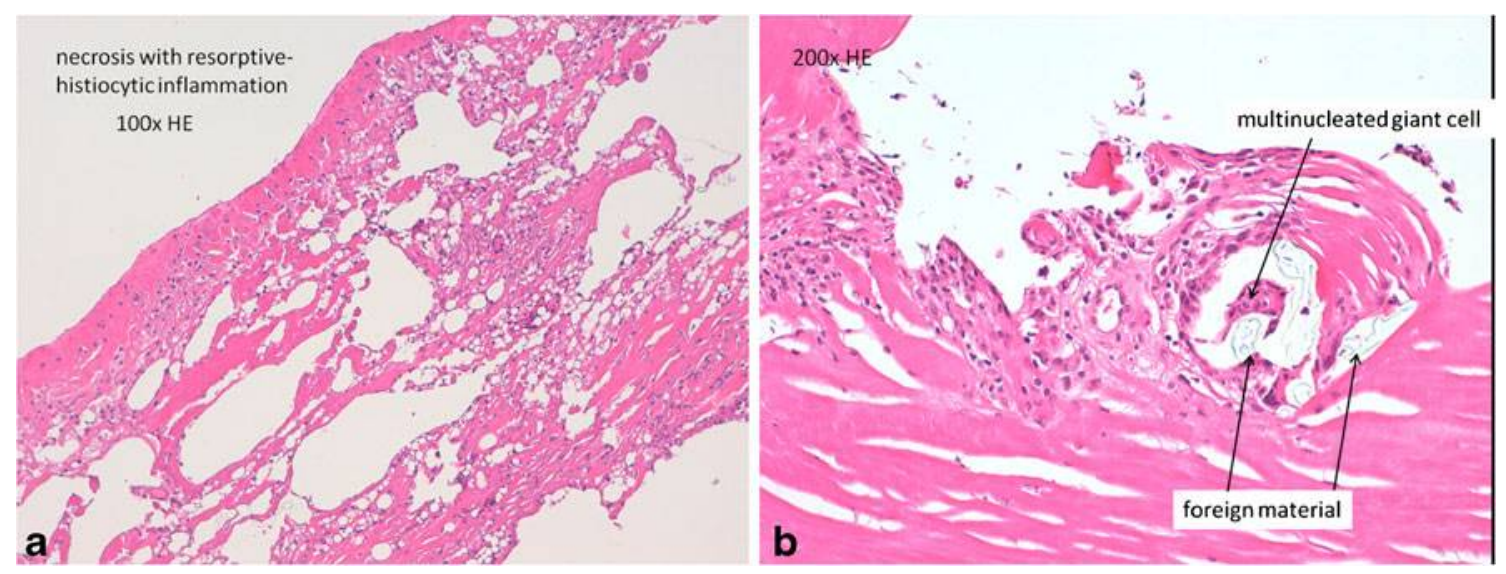

Fig. 6 Histology of patient B showing necrosis with resorptive histiocytic inflammation (a) and formation of granulomas with multinucleated giant cells around foreign material (b)

There were enlarged axillary lymph nodes on the same side, which also showed a strong silicone signal.

Ultrasound images had been acquired at initial presentation and were available for correlation in both patients. There was no sign of rupture in patient $\mathrm{A}$, whose implants appeared intact on CT. In patient B with the suspected rupture, ultrasound showed a "stepladder" sign of the implant and a "snowstorm" sign in the axillary lymph node on the same side (Fig. 4). With the ultrasound and CT findings suggesting implant rupture, patient $\mathrm{B}$ then agreed to MRI with sedation for surgical planning. MRI also indicated rupture of the left implant with "linguine" and "salad oil" signs in the implant and strong signal in the axillary lymph node in silicone-specific sequences (Fig. 5).

Surgery confirmed the findings in both patients. The Mentor prostheses of patient A were explanted without complication. In patient B with PIP implants, the left implant was very fragile and completely disintegrated at explantation. The right implant was found intact and explanted without complications. Adhering to current pertinent guidelines [25], the axillary lymph nodes were not sampled or resected.

In patient $\mathrm{B}$, histology confirmed silicone outside the implant with the formation of granulomas in the tissue medial and inferior of the implant (Fig. 6).

\section{Discussion}

The diagnostic evaluation of the breast after augmentation remains a challenge in several respects. MRI is obviously the technique of choice but is time consuming and expensive and may yield a substantial number of false-positive results in a cancer screening setting. Also, there is a small number of patients in whom contraindications or claustrophobia curtail its use. In the literature, it is also being discussed controversially whether general health insurance should pay for more expensive screening techniques or even for the follow-up of the implant after an augmentation for cosmetic purposes [1, 26-28].

Based on our initial results, it seems that dual-energy CT might become an option for implant evaluation in particular cases, especially if there are contraindications for MRI. Of course, in contrast to emerging high-resolution breast CT [29], the technique is not sufficient to simultaneously screen for cancer and implies exposure to ionising radiation. The equivalent dose of 2.2 and $2.7 \mathrm{mSv}$ in our patients was well below the reference limit for chest examinations (DLP $\left.400 \mathrm{mGy}^{*} \mathrm{~cm}[30]\right)$. In conventional mammography, patients with implants receive higher radiation doses owing to the density of the implants, amounting to some $10.7 \mathrm{mGy}$ per breast or a total equivalent dose of $2.6 \mathrm{mSv}$ [31, 32], so the overall dose is comparable. Thus, weighing the health risks of a ruptured implant against the low risk of radiationinduced cancer, the indication may appear similar as in many other diseases that are routinely evaluated by CT. A strength of the technique is the specific depiction of silicone without the administration of contrast material, so there are no other side effects or risks. The diagnostic value of the two initial patient examinations, clearly showing a strong silicone signal and indicating implant rupture and silicone in the axillary lymph nodes in one of the patients, seems to justify the use of this technique.

In conclusion, dual-energy $\mathrm{CT}$ offers the possibility of specifically visualising silicone, making it feasible to evaluate breast implants for rupture. Thus, dual-energy CT may serve as an alternative technique, especially for patients with contraindications for MRI. A systematic clinical trial will be required to determine the diagnostic accuracy of this technique and to define appropriate indications.

Acknowledgments Bernhard Krauss is an employee of Siemens Healthcare. 


\section{References}

1. Smith R, Lunt N, Hanefeld J (2012) The implications of PIP are more than just cosmetic. Lancet 379:1180-1181

2. AFSSAPS (2012) Letter to healthcare professionnals - Important information on the monitoring of women who have breast implants prefilled with PIP silicone gel. Available via http://ansm.sante.fr/ var/ansm_site/storage/original/application/cc0700f9c3a85bb c1e9496eb2f9c4f4b.pdf. Accessed 28 Aug 2012

3. Yang N, Muradali D (2011) The augmented breast: a pictorial review of the abnormal and unusual. AJR Am J Roentgenol 196: W451-W460

4. Goodman CM, Cohen V, Thornby J, Netscher D (1998) The life span of silicone gel breast implants and a comparison of mammography, ultrasonography, and magnetic resonance imaging in detecting implant rupture: a meta-analysis. Ann Plast Surg 41:577585 , discussion $585-576$

5. Hammond DC (2012) Discussion: prevalence of rupture in poly implant prothese silicone breast implants, recalled from the European market in 2010. Plast Reconstr Surg 129:1379-1380

6. Maijers MC, Niessen FB (2012) Prevalence of rupture in poly implant prothese silicone breast implants, recalled from the European market in 2010. Plast Reconstr Surg 129:1372-1378

7. Aktouf A, Auquit-Auckbur I, Coquerel-Beghin D, Delpierre V, Milliez PY (2012) Breast augmentation by poly implant protheses silicone implants: retrospective study about 99patients. Rupture analysis and management. Ann Chir Plast Esthet. doi:10.1016/ j.anplas.2012.04.007

8. SCENIHR (2012) Scientific Committee on Emerging and Newly Identified Health Risks: Safety of PIP Silicone Breast Implant. Available via http://ec.europa.eu/health/scientific_committees/ emerging/docs/scenihr o 034.pdf. Accessed 28 Aug 2012

9. Venkataraman S, Hines $\bar{N}$, Slanetz PJ (2011) Challenges in mammography: part 2, multimodality review of breast augmentationimaging findings and complications. AJR Am J Roentgenol 197: W1031-W1045

10. Cilotti A, Marini C, Iacconi C et al (2006) Ultrasonographic appearance of breast implant complications. Ann Plast Surg $56: 243-247$

11. Venta LA, Salomon CG, Flisak ME, Venta ER, Izquierdo R, Angelats J (1996) Sonographic signs of breast implant rupture. AJR Am J Roentgenol 166:1413-1419

12. Caskey CI, Berg WA, Anderson ND, Sheth S, Chang BW, Hamper UM (1994) Breast implant rupture: diagnosis with US. Radiology 190:819-823

13. Di Benedetto G, Cecchini S, Grassetti L et al (2008) Comparative study of breast implant rupture using mammography, sonography, and magnetic resonance imaging: correlation with surgical findings. Breast J 14:532-537

14. Ikeda DM, Borofsky HB, Herfkens RJ, Sawyer-Glover AM, Birdwell RL, Glover GH (1999) Silicone breast implant rupture: pitfalls of magnetic resonance imaging and relative efficacies of magnetic resonance, mammography, and ultrasound. Plast Reconstr Surg 104:2054-2062
15. Weizer G, Malone RS, Netscher DT, Walker LE, Thornby J (1995) Utility of magnetic resonance imaging and ultrasonography in diagnosing breast implant rupture. Ann Plast Surg 34:352-361

16. Reynolds HE, Buckwalter KA, Jackson VP, Siwy BK, Alexander SG (1994) Comparison of mammography, sonography, and magnetic resonance imaging in the detection of silicone-gel breast implant rupture. Ann Plast Surg 33:247-255, discussion 256-247

17. Ahn CY, Shaw WW, Narayanan K et al (1993) Definitive diagnosis of breast implant rupture using magnetic resonance imaging. Plast Reconstr Surg 92:681-691

18. Gorczyca DP, Gorczyca SM, Gorczyca KL (2007) The diagnosis of silicone breast implant rupture. Plast Reconstr Surg 120:49S$61 \mathrm{~S}$

19. Holmich LR, Vejborg I, Conrad C, Sletting S, McLaughlin JK (2005) The diagnosis of breast implant rupture: MRI findings compared with findings at explantation. Eur J Radiol 53:213-225

20. Madhuranthakam AJ, Smith MP, Yu H et al (2012) Water-silicone separated volumetric MR acquisition for rapid assessment of breast implants. J Magn Reson Imaging 35:1216-1221

21. Koh DM, Blackledge M, Burns S et al (2012) Combination of chemical suppression techniques for dual suppression of fat and silicone at diffusion-weighted MR imaging in women with breast implants. Eur Radiol. doi:10.1007/s00330-012-2531-1

22. Johnson TR, Krauss B, Sedlmair M et al (2007) Material differentiation by dual energy CT: initial experience. Eur Radiol 17:1510 1517

23. Choi JJ, Lee JH, Kang BJ et al (2010) Clinical and imaging characteristics of Polyimplant Prosthesis hydrogel breast implants. J Comput Assist Tomogr 34:449-455

24. Huda W, Ogden KM, Khorasani MR (2008) Converting doselength product to effective dose at CT. Radiology 248:995-1003

25. DGGG (2012) Empfehlungen für Fachärzte zum Umgang mit Brustimplantaten der Firma PIP und Rofil. Available via http:// www.dggg.de/fileadmin/public_docs/Stellungnahmen/2012-0111-DGGG-DGS-DGPW-Brustimplantate.pdf. Accessed 28 Aug 2012

26. Torjesen I (2012) NHS patients are choosing to have PIP implants removed whether or not they have burst. BMJ 344:e1460

27. O'Dowd A (2012) Around 1000 women with private sector PIP implants seek NHS help. BMJ 344:e972

28. O'Dowd A (2012) Government puts pressure on private sector to pay for removal of PIP breast implants. BMJ 344:e249

29. Kalender WA, Beister M, Boone JM, Kolditz D, Vollmar SV, Weigel MC (2012) High-resolution spiral CT of the breast at very low dose: concept and feasibility considerations. Eur Radiol 22:1-8

30. BFS (2010) Bundesamt für Strahlenschutz: Bekanntmachung der aktualisierten diagnostischen Referenzwerte für diagnostische und interventionelle Röntgenuntersuchungen. Available via www.bfs.de/ de/ion/medizin/referenzwerte02.pdf. Accessed 28 Aug 2012

31. ICRP (2007) The 2007 Recommendations of the International Commission on Radiological Protection. ICRP publication 103. Ann ICRP 37:1-332

32. Smathers RL, Boone JM, Lee LJ, Berns EA, Miller RA, Wright AM (2007) Radiation dose reduction for augmentation mammography. AJR Am J Roentgenol 188:1414-1421 